04,05

\title{
Теплоемкость легированного эрбием галлий-гадолиниевого граната
}

\author{
(C) И.Е. Лезова ${ }^{1}$, Е.В. Шевченко ${ }^{1}$, Е.В. Чарная ${ }^{1}$, Е.Н. Хазанов ${ }^{2}$, А.В. Таранов ${ }^{2}$ \\ ${ }^{1}$ Санкт-Петербургский государственный университет, \\ Санкт-Петербург, Россия \\ ${ }^{2}$ Институт радиотехники и электроники им. В.А. Котельникова РАН, \\ Москва, Россия \\ E-mail: irina_gospodchikova@mail.ru
}

(Поступила в Редакцию 24 апреля 2018 г.)

\begin{abstract}
Приведены результаты исследования теплоемкости в легированном эрбием кристалле галлийгадолиниевого граната в сравнении с данными для нелегированного граната. Измерения проводились в температурном диапазоне от 1.9 до $220 \mathrm{~K}$ в магнитных полях от 0 до 9Т. Температурные зависимости теплоемкости интерпретировались с учетом вкладов Шоттки, обусловленных ионами $\mathrm{Gd}^{3+}$ и $\mathrm{Er}^{3+}$, и вкладов тепловых колебаний кристаллической решетки. Рассчитаны величины энтропии и магнитной энтропии.
\end{abstract}

Работа выполнена при поддержке РФФИ (гранты № 16-07-00181 и 18-07-00191).

DOI: $10.21883 /$ FTT.2018.10.46516.115

\section{1. Введение}

Галлий-гадолиниевые гранаты $\mathrm{Gd}_{3} \mathrm{Ga}_{5} \mathrm{O}_{12}(\mathrm{GGG})$ относятся к наиболее известным и широко используемым редкоземельным гранатам [1]. В основном, монокристаллы GGG применяются в качестве оптических и магнито-оптических элементов, подложек и в ювелирной промышленности. Кристаллы GGG были также предложены в качестве материалов для магнитных рефрижераторов [2] благодаря значительной величине магнитной энтропии. В ряде более поздних исследований было показано, что различные варианты замешения в решетке GGG как ионов гадолиния, так и ионов галлия могут приводить к некоторому усилению магнитокалорического эффекта [3-6]. В связи с возможным использованием чистых и легированных кристаллов GGG для охлаждения методом адиабатического размагничивания значительное внимание уделялось измерениям их теплоемкости при низких температурах $[3,4,7]$. При этом, однако, не проводилась количественная интерпретация температурных зависимостей теплоемкости с учетом вкладов колебаний кристаллической решетки и штарковских уровней парамагнитных ионов (аномалий Шоттки). В настоящей работе приводятся результаты экспериментальных исследований теплоемкости кристаллов GGG с примесью эрбия (GGG:Er) в нулевом поле и внешних полях до 9Т в сравнении с данными для чистых GGG гранатов, а также теоретическая интерпретация полученных результатов. Отметим, что измерения теплоемкости кристаллов GGG, легированных эрбием, ранее не проводились.

\section{2. Образцы и эксперимент}

Чистый и легированный эрбием монокристаллы GGG были выращены методом горизонтальной направленной кристаллизации в молибденовом контейнере. Концентрация эрбия в легированном кристалле составляла 5 at.\% от количества гадолиния в стехиометрическом GGG. Образцы для исследования теплоемкости были вырезаны из средних частей буль. Они имели вид пластин, ориентированных перпендикулярно кубической оси кристаллов, с толщиной около $0.3 \mathrm{~mm}$ и поперечным сечением около $20 \mathrm{~mm}^{2}$.

Кристаллы гранатов имеют пространственную симметрию $I a \overline{3} d$. Трехвалентные ионы гадолиния $\mathrm{Gd}^{3+}$, в основном, занимают додекаэдрические $c$-позиции в кристаллической решетке. При слабом легировании трехвалентные ионы эрбия $\mathrm{Er}^{3+}$ замещают ионы гадолиния. Элементарная ячейка содержит 8 формульных единиц. Фазовый переход в антиферромагнитное упорядоченное состояние в кристаллах GGG не был обнаружен вплоть до $25 \mathrm{mK}$ [8], несмотря на то, что парамагнитная температура Кюри-Вейсса приблизительно равна $-2 \mathrm{~K}$. Подавление антиферромагнитного фазового перехода наблюдается во всех гранатах с парамагнитными ионами и обусловлено особенностью кристаллической решетки, в которой додекаэдрические позиции образуют гиперкагоме структуру.

Теплоемкость измерялась на установке PPMS9 + Ever-Cool-II производства Quantum Design с использованием встроенной опции. Измерения проводились в температурном диапазоне $1.9-220 \mathrm{~K}$ в магнитных полях от 0 до 9 T.

\section{3. Результаты и обсуждение}

Температурные зависимости теплоемкости в различных магнитных полях показаны на рис. 1 для кристаллов GGG: $\operatorname{Er}(a)$ и чистого GGG $(b)$. Видно, что магнитное поле оказывает заметное влияние на теплоемкость при температурах ниже $80 \mathrm{~K}$. Вклад в теплоемкость анома- 

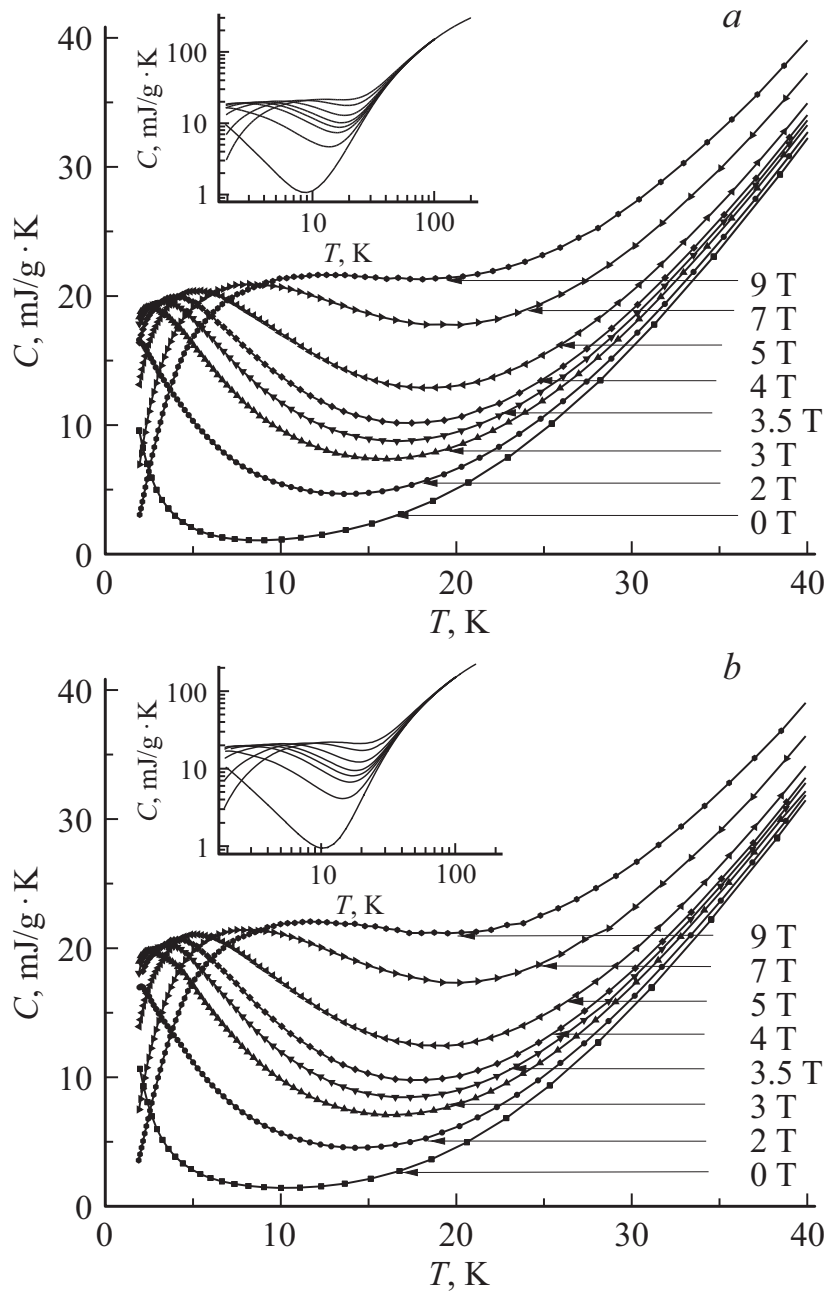

Pис. 1. Температурные зависимости теплоемкости для GGG: $\operatorname{Er}(a)$ и GGG $(b)$ ниже $40 \mathrm{~K}$ в различных магнитных полях, указанных на рисунке. На вставках показана теплоемкость в тех же магнитных полях во всем температурном диапазоне от 1.9 до $220 \mathrm{~K}$ в двойном логарифмическом масштабе.

лий Шоттки, обусловленных ионами $\mathrm{Gd}^{3+}$ и $\mathrm{Er}^{3+}[9]$, доминирует ниже $30 \mathrm{~K}$. С увеличением магнитного поля вид аномалий Шоттки изменяется за счет сдвига и расщепления уровней энергии ионов в кристаллическом поле. Выше $30 \mathrm{~K}$ превалирует решеточный (фононный) вклад [9].

Численный анализ теплоемкости диэлектрических кристаллов с парамагнитными ионами основывается на учете нескольких независимых вкладов: пиков теплоемкости, вызываемых магнитными или структурными фазовыми переходами, фононной теплоемкости, представляемой в виде суммы вкладов Дебая и Эйнштейна, и аномалий Шоттки [9]. Галлий-гадолиниевые гранаты не претерпевают структурных фазовых переходов вплоть до плавления. Магнитная фазовая диаграмма кристаллов GGG является необычной и богатой, причем дальний антиферромагнитный порядок формируется только при приложении внешних магнитных полей, лежащих в определенном диапазоне [10-12]. Однако магнитные особенности наблюдаются значительно ниже $1.9 \mathrm{~K}$, так что они не сказываются на измерениях теплоемкости, проведенных в настоящей работе. Таким образом, аппроксимация температурных зависимостей теплоемкости должна включать вклады решеточных колебаний и аномалий Шоттки.

Теплоемкость Дебая одного моля вещества при температуре $T$ записывается в виде

$$
C_{D}(T)=3 r_{D} R\left(\frac{T}{\theta}\right)^{3} \int_{0}^{\frac{\theta}{T}} \frac{x^{4} e^{x}}{\left(e^{x}-1\right)^{2}} d x
$$

где $r_{D}$ - число колебательных мод, интерпретируемых в рамках модели Дебая, $R$ - газовая постоянная, $\theta-$ температура Дебая. Вклад Эйнштейна в расчете на моль вещества имеет вид

$$
C_{E}(T)=r_{E} R\left(\frac{\theta_{E}}{T}\right)^{2} \frac{e^{\frac{\theta_{E}}{T}}}{\left(e^{\frac{\theta_{E}}{T}}-1\right)^{2}},
$$

где $\theta_{E}-$ температура Эйнштейна и $r_{E}-$ число колебательных мод, рассматриваемых в рамках этой модели.

Аномалии Шоттки связаны с уровнями Штарка парамагнитных ионов в кристаллическом поле. Вклад Шоттки записывается в виде [9]

$$
C_{s}=\frac{n R}{T^{2}}\left[\frac{\sum_{i} \Delta_{i}^{2} g_{i} e^{-\frac{\Delta_{i}}{T}}}{g_{0}+\sum_{i} g_{i} e^{-\frac{\Delta_{i}}{T}}}-\left(\frac{\sum_{i} \Delta_{i} g_{i} e^{-\frac{\Delta_{i}}{T}}}{g_{0}+\sum_{i} g_{i} e^{-\frac{\Delta_{i}}{T}}}\right)^{2}\right],
$$

где $n$ - число парамагнитных ионов определенного вида в молекулярной формуле, $\Delta_{i}$ - разность энергий в $\mathrm{K}$ между основным уровнем и возбужденным уровнем с номером $i, g_{0}$ и $g_{i}-$ степень вырождения основного и возбужденного состояний, $i=1$ соответствует нижнему возбужденному уровню.

Для анализа вклада Шоттки в теплоемкость чистого кристалла GGG необходимо знать структуру уровней ионов $\mathrm{Gd}^{3+}$. Согласно [13] нижним является спиновый мультиплет ${ }^{8} S_{7 / 2}$. Остальные уровни энергии отстоят более, чем на $30000 \mathrm{~cm}^{-1}$ от основного мультиплета. Нижний мультиплет слабо взаимодействует с кристаллическим полем. Теоретические оценки [13] показывают, что восьмикратно вырожденное нижнее состояние расщепляется на четыре крамерсовских дублета с энергиями $0,0.3,0.5$ и $0.6 \mathrm{~cm}^{-1}$ (соответственно $0,0.43,0.72$ и $0.86 \mathrm{~K})$.

Уровни энергии низколежащих состояний трехвалентного эрбия в галлий-гадолиниевом гранате были экспериментально определены в работе [14]. Основной мультиплет ${ }^{4} I_{15 / 2}$ расщепляется на 8 крамерсовских дублетов. Четыре нижних дублета имеют энергии 0,31 , 44 и $63 \mathrm{~cm}^{-1}(0,44.6,63.3$ и $90.6 \mathrm{~K})$, остальные уровни 
лежат выше $380 \mathrm{~K}$. Отметим, что три нижних возбужденных дублета имею значения энергии близкие к крамерсовским состояниям эрбия в гранатах $\mathrm{Y}_{3} \mathrm{Sc}_{2} \mathrm{Ga}_{3} \mathrm{O}_{12}$ [15] и $\mathrm{Y}_{3} \mathrm{Al}_{5} \mathrm{O}_{12}$ [16]. Недавно, однако, было показано, что для численной интерпретации результатов измерений теплоемкости в смешанных алюмо-эрбиевых гранатах необходимо предположить дополнительное расщепление нижнего крамерсовского дублета иона $\mathrm{Er}^{3+}$ магнитными полями от соседей $[17,18]$. Это дополнительное расщепление на два синглета $\Delta_{\mathrm{Er}}^{\prime}$ имело порядок величины $1 \mathrm{~K}$.

Рассмотрим эволюцию теплоемкости с температурой в отсутствие внешнего магнитного поля. Мы ограничимся аппроксимацией экспериментальных зависимостей ниже $40 \mathrm{~K}$, где, как показано дальше, является достаточным учет всего двух вкладов Эйнштейна. Температура Дебая для обоих чистого и легированного эрбием гранатов была принята равной $500 \mathrm{~K}[19,20]$. При расчете вкладов Эйнштейна температуры $\theta_{E 1}$ и $\theta_{E 2}$ играли роль подгоночных параметров, как и коэффициенты $r_{D}, r_{E 1}$ и $r_{E 2}$. Для чистого кристалла GGG учитывался вклад Шоттки от ионов гадолиния. В качестве подгоночных параметров выступали разности энергий $\Delta_{i}(i=1,2,3)$ между возбужденными и основным крамерсовскими дублетами. Для легированного граната учитывался вклад ионов эрбия. Величина дополнительного расщепления нижнего крамерсовского дублета за счет локальных магнитных полей слабо влияла на качество подгонки, поэтому $\Delta_{\mathrm{Er}}^{\prime}$ принималось равным $1 \mathrm{~K}$, таким же, как в [18]. Энергии остальных крамерсовских дублетов принимались равными значениям, приведенным в [14].

Результаты аппроксимации представлены на рис. 2. На нем показаны как отдельные вклады в теплоемкость, так и их сумма. Из рис. 2 видно, что при низких температурах теплоемкость практически полностью определяется аномалиями Шоттки. Значения всех подгоночных параметров собраны в таблице. Следует подчеркнуть, что расчетные величины расщепления основного мультиплета трехвалентных ионов гадолиния почти на порядок превышают теоретические оценки. Можно предположить, что в расщеплении играют значительную роль магнитные поля от соседних парамагнитных ионов гадолиния, как было показано в случае алюмо-эрбиевых гранатов $[17,18]$. Из таблицы видно, что легирование эрбием приводит к слабым изменениям подгоночных параметров для теплоемкости, однако даже при низкой

Коэффициенты $r_{D}, r_{E 1}, r_{E 2}$ в соотношениях $(1,2)$, соответствующие температуры Эйнштейна $\theta_{E 1}$ и $\theta_{E 2}$ и расщепления основного мультиплета ионов гадолиния на четыре крамерсовских дублета $\Delta_{1}, \Delta_{2}$ и $\Delta_{3}$

\begin{tabular}{c|c|c|c|c|c|c|c|c}
\hline Sample & $r_{D}$ & $\theta_{E 1}(\mathrm{~K})$ & $r_{E 1}$ & $\theta_{E 2}(\mathrm{~K})$ & $r_{E 2}$ & $\Delta_{1}(\mathrm{~K})$ & $\Delta_{2}(\mathrm{~K})$ & $\Delta_{3}(\mathrm{~K})$ \\
\hline GGG & 54.6 & 126 & 3 & 230 & 2.4 & 1.5 & 2.4 & 4.4 \\
GGG : $E r$ & 54.6 & 127 & 3 & 210 & 2.4 & 1.5 & 2.4 & 4.2
\end{tabular}
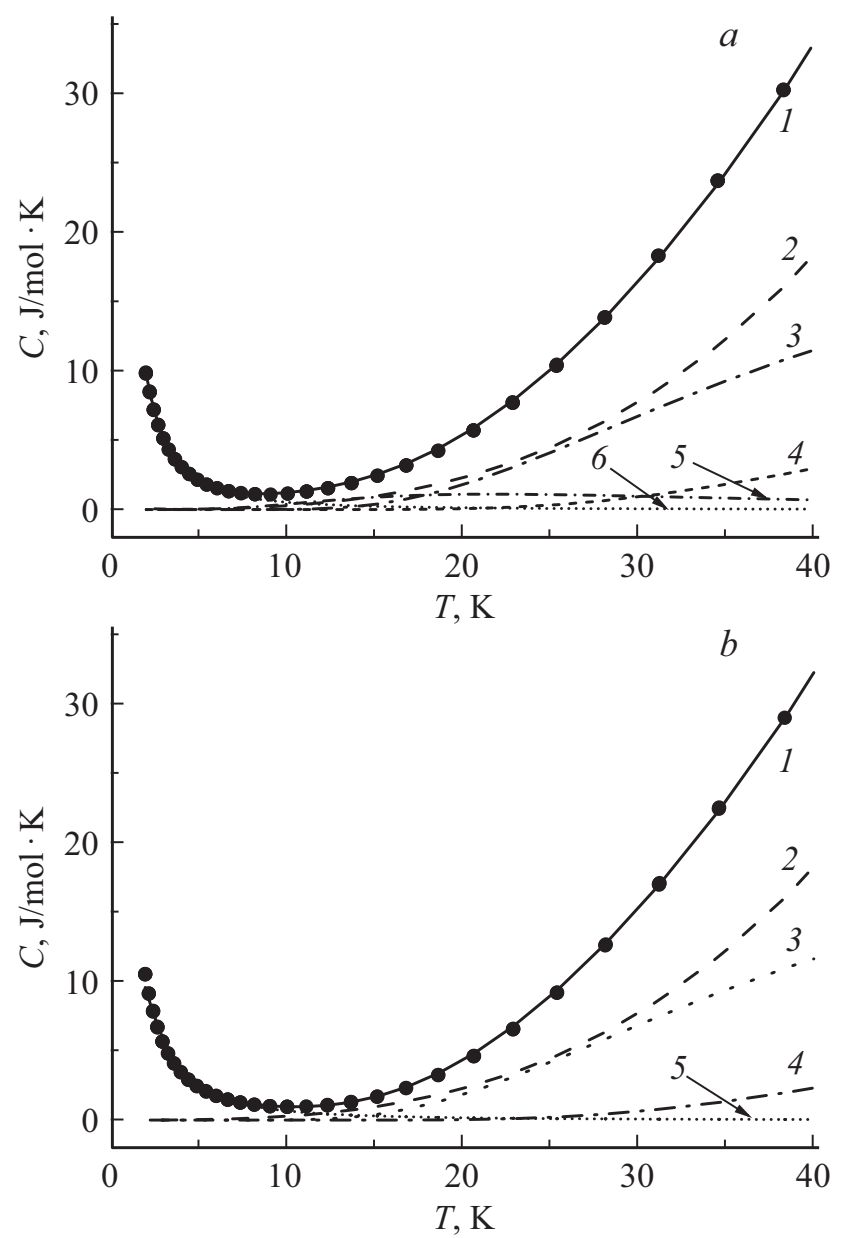

Pис. 2. Аппроксимация теплоемкости в кристаллах GGG: $\mathrm{Er} \quad(a)$ и $\mathrm{GGG} \quad(b)$ ниже $40 \mathrm{~K}$. Кружки экспериментальные данные. Сплошные линии показывают полную рассчитанную теплоемкость (1), вклад Дебая (2), вклады Эйнштейна $(3,4)$ и аномалии Шоттки, индуцированные ионами $\mathrm{Gd}(5)$ и $\operatorname{Er}(6)$.

концентрации эрбия вклад Шоттки, обусловленный наличием эрбия, является заметным.

Приложение внешнего магнитного поля вызывает расщепление крамерсовских дублетов, которое должно быть пропорционально полю. В результате подьем теплоемкости с понижением температуры становится сначала более выраженным, а затем на кривых теплоемкости появляются максимумы, которые сдвигаются в сторону высоких температур с дальнейшим возрастанием поля. Зависимости положения максимумов теплоемкости от поля для легированного и нелегированного кристаллов GGG показаны на рис. 3. Как и ожидалось, сдвиг максимума теплоемкости приблизительно линейно зависит от приложенного поля. Однако величины сдвига значительно превышают оценки, сделанные с учетом значения $g$-фактора для ионов гадолиния, соответствующего нижнему спиновому мультиплету $(g=2)$, а также данным электронного парамагнитного резонанса в чистом GGG [21]. Сильное отличие расщепле- 
ния уровней по данным теплоемкости от спектроскопических данных требует проведения дополнительных исследований.

Проведенные измерения теплоемкости во внешних магнитных полях позволяют рассчитать изменение энтропии и магнитной энтропии от $1.9 \mathrm{~K}$ до некоторой температуры $T$. В том случае, когда вклад в энтропию ниже $1.9 \mathrm{~K}$ оказывается незначительным, расчет изменения энтропии дает хорошее приближение для оценки как полной энтропии, так и полной магнитной энтропии при $T>1.9 \mathrm{~K}$. Однако в чистом и легированном кристаллах GGG рост теплоемкости при понижении температуры ниже $10 \mathrm{~K}$ в нулевом и слабых магнитных полях указывает на значительную роль низкотемпературного вклада

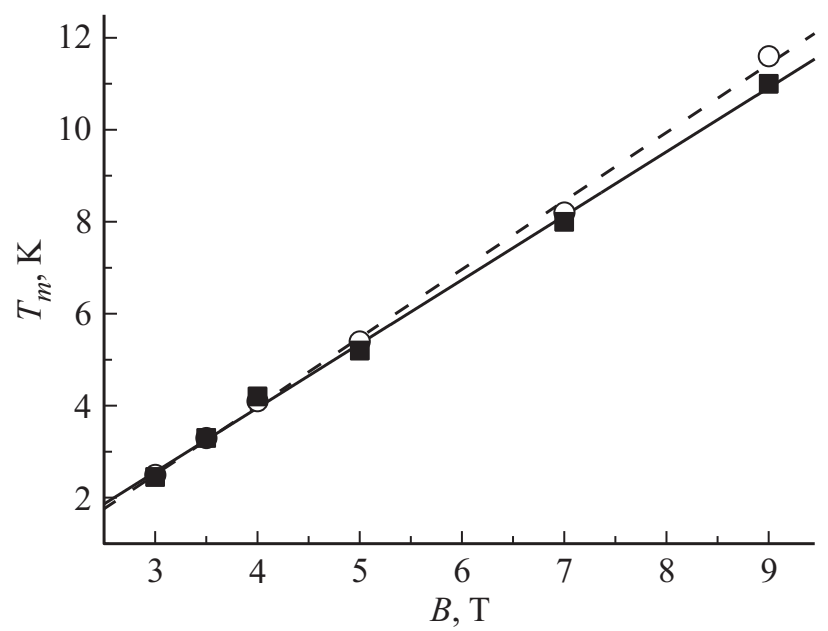

Pис. 3. Зависимости температуры максимумов теплоемкости $T_{m}$ от магнитного поля для кристаллов GGG: $\operatorname{Er}$ (открытые символы) и GGG (заполненные символы). Прямые линии показывают линейные аппроксимации.

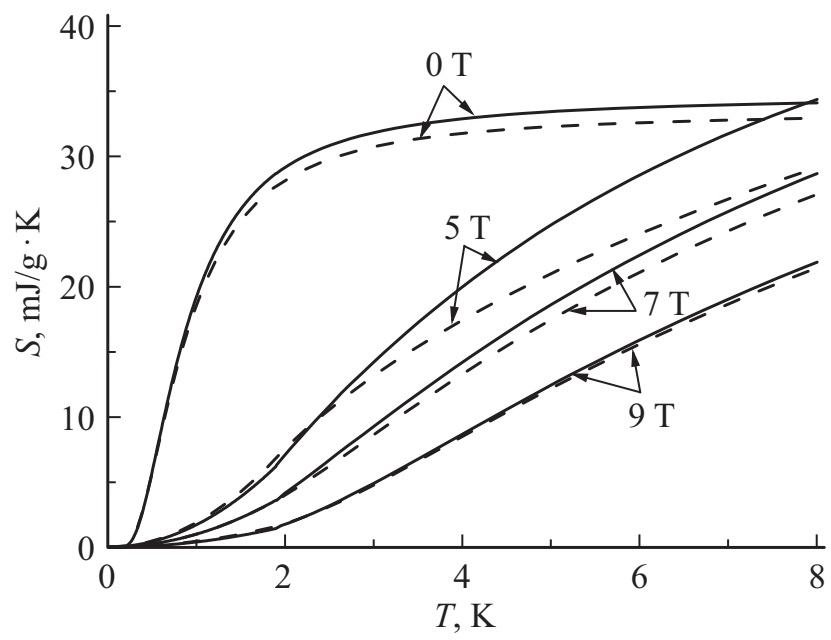

Рис. 4. Результаты расчета температурных зависимостей энтропии до $8 \mathrm{~K}$ для кристаллов GGG: $\operatorname{Er}$ (штриховые линии) и GGG (сплошные линии) при магнитных полях, указанных на рисунке.

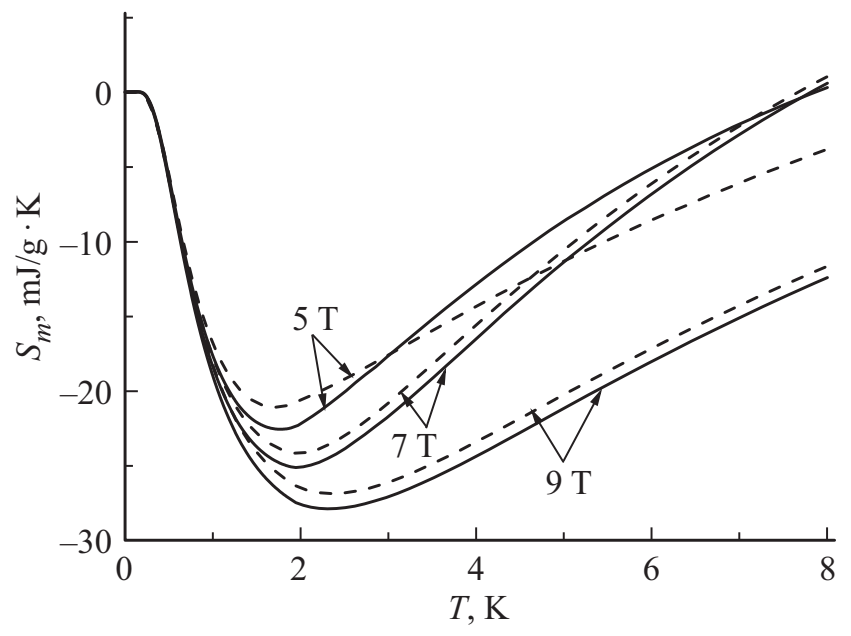

Рис. 5. Рассчитанная магнитная энтропия для кристаллов GGG:Er (штриховые линии) и GGG (сплошные линии) при магнитных полях, указанных на рисунке.

в энтропию. Поэтому для оценки увеличения энтропии в интервале от 0 до $1.9 \mathrm{~K}$ можно использовать теоретические зависимости, полученные выше, хотя такие оценки и не включают вклады короткодействующих и дальнодействующих магнитных корреляций.

Результаты расчета температурных зависимостей энтропии до $8 \mathrm{~K}$ представлены на рис. 4. Энтропия в нулевом поле рассчитывалась по формуле

$$
S(T)=\int_{0}^{T} \frac{C_{0}(t)}{t} d t .
$$

где $C_{0}(t)$-- теоретическая теплоемкость при текущей температуре $t$. Аналогичным образом находилась энтропия $S_{B}(T)$ в поле $B$. Магнитный вклад в энтропию рассчитывался как разница энтропий в поле и в отсутствие поля $S_{m}(T)=S_{B}(T)-S(T)$. Магнитная энтропия для трех полей показана на рис. 5. Видно, что слабое легирование эрбием приводит к изменениям энтропии, сравнимым с влиянием значительного замещения галлия алюминием [5]. Отметим, что в диапазоне от 0 до $8 \mathrm{~K}$ магнитная энтропия отрицательна в чистом и легированном GGG. При этом легирование эрбием наиболее существенно сказывается на величине магнитной энтропии для полей около $5 \mathrm{~T}$.

\section{4. Заключение}

Экспериментальная теплоемкость чистого и легированного эрбием кристаллов GGG аппроксимирована в температурном интервале $1.9-40 \mathrm{~K}$ суммой вкладов аномалий Шоттки, обусловленных ионами гадолиния и эрбия, и решеточных вкладов, рассчитываемых в рамках моделей Дебая и Эйнштейна. Показано, что расщепления основного спинового мультиплета гадолиния имеют величины, на порядок превышающие теоретические 
оценки. Для легированного эрбием кристалла продемонстрирован существенный вклад Шоттки от ионов эрбия. Рассчитана энтропия и магнитная энтропия с использованием проведенного анализа теплоемкости. Показано, что даже слабое легирование эрбием приводит к заметным изменениям энтропии.

Авторы благодарят А.А. Каминского за предоставление кристаллов для исследования. Измерения проводились на оборудовании Ресурсного Центра „Центр диагностики функциональных материалов для медицины фармакологии и наноэлектроники“, Научный парк СПбГУ.

\section{Список литературы}

[1] А.А Каминский. Лазерные кристаллы. Наука, М. (1975). $356 \mathrm{c}$.

[2] Y. Hakuraku, H. Ogata. J. Appl. Phys. 60, 3266 (1986).

[3] T. Numazawa, K. Kamiya, T. Okano, K. Matsumoto. Physica B 329, 1656 (2003).

[4] K. Matsumoto, A. Matsuzakiy, K. Kamiyaz, T. Numazawa. Jpn. J. Appl. Phys. 48, 113002 (2009).

[5] A.C. Sackville Hamilton, G.I. Lampronti, S.E. Rowley, S.E. Dutton. J. Phys.: Condens. Matter 26, 116001 (2014).

[6] C.P. Reshmi, S. SavithaPillai, K.G. Suresh, M.R. Varma. J. Magn. Magn. Mater. 3241962 (2012).

[7] D.G. Onn, H. Meyer, J.P. Remeika. Phys. Rev. B 156, 663 (1967).

[8] S.R. Dunsiger, J.S. Gardner, J.A. Chakhalian, A.L. Cornelius, M. Jaime, R.F. Kiefl, R. Movshovich, W.A. MacFarlane, R.I. Miller, J.E. Sonier, B.D. Gaulin. Phys. Rev. Lett. 85, 3504 (2000).

[9] A. Tari. The specific heat of matter at low temperatures. Imperial College Press, London (2003). 335 c.

[10] P. Schiffer, A.P. Ramirez, D.A. Huse, A.J. Valentino. Phys. Rev. Lett. 73, 2500 (1994).

[11] Y.K. Tsui, N. Kalechofsky, C.A. Burns, P. Schiffer. J. Appl. Phys. 85, 4512 (1999).

[12] J.A. Quilliam, S. Meng, H.A. Craig, L.R. Corruccini, G. Balakrishnan, O.A. Petrenko, A. Gomez, S.W. Kycia, M.J.P. Gingras, J.B. Kycia. Phys. Rev. B 87, 174421 (2013).

[13] J.B. Gruber, M.E. Hills, C.A. Morrison, G.A. Turner, M.R. Kokta. Phys. Rev. B 37, 8564 (1988).

[14] J.B. Gruber, D.K. Sardar, B. Zandi, J.A. Hutchinson, C.W. Trussell. J. Appl. Phys. 93, 3137 (2003).

[15] J.B. Gruber, J.R. Quagliano, M.F. Reid, F.S. Richardson, M.E. Hills, M.D. Seltzer, S.B. Stevens, C.A. Morrison, T.H. Allik. Phys. Rev. B 48, 15561 (1993).

[16] G.W. Burdick, J.B. Gruber, K.L. Nash, S. Chandra, D.K. Sardar. Spectrosc. Lett. 43, 406 (2010).

[17] A. Kushino, Y. Aoki, N.Y. Yamasaki, T. Namiki, Y. Ishisaki, T.D. Matsuda, T. Ohashi, K. Mitsuda, T. Yazawa. J. Appl. Phys. 90, 5812 (2001).

[18] E.V. Shevchenko, E.V. Charnaya, E.N. Khazanov, A.V. Taranov, A.S. Bugaev. J. Alloy Compd. 717, 183 (2017).
[19] E. Langenberg, E. Ferreiro-Vila, V. Leborán, A.O. Fumega, V. Pardo, F. Rivadulla. APL Mater. 4, 104815 (2016).

[20] W. Dait, E. Gmelin, R. Kremer. J. Phys. D 21, 628 (1988).

[21] J. Barak, M.X. Huang, S.M. Bhagat. J. Appl. Phys. 71, 849 (1992).

Редактор Т.Н. Василевская 